\title{
Effects of goodness and other properties of patterns on discriminative performance of monkeys
}

\author{
ALLAN M. SCHRIER, MORRIS L. POVAR, and JUDITH E. SCHRIER \\ Brown University, Providence, Rhode Island 02912
}

\begin{abstract}
Stumptailed monkeys were given a series of pattern discrimination problems of the type used in studies with humans to test Garner's information-theoretic theory of figural goodness. Unlike humans', the monkeys' learning and response latency were not faster for problems involving good patterns (coming from small rotation-and-reflection equivalence sets) than for problems involving poor patterns (coming from large rotation-and-reflection equivalence sets). Like humans, the monkeys found problems involving patterns from the same equivalence set, regardless of the size of the set, to be much harder to learn than problems involving patterns from different sets. Learning was faster, the greater the number of unique elements distinguishing a pair of patterns. Effects of specific types of reflection and rotation were also analyzed. The results suggest that there are both similarities and differences in the processing of patterns by monkeys and humans, and that the processing can, at the same time, involve both whole patterns and elements or features of patterns.
\end{abstract}

Gestalt psychologists emphasized that figures cannot be specified solely in terms of their elements, but also have organization or "goodness," and that figures differ in amount of goodness. Garner (1962), in attempting to quantify goodness, proposed that a pattern may be perceived as one of a set of different, but alternative or equivalent, patterns; the greater the size of a pattern's "equivalence set," the greater is the uncertainty connected with perceiving the pattern. Garner further hypothesized that pattern goodness is inversely related to the size of the equivalence set, and that, even when only one member of the set is presented, processing occurs with reference to an inferred set.

These assumptions have been tested in a series of studies, many of which involved patterns of five dots in a 3 by 3 matrix first used by Garner and Clement (1963). Equivalence sets were established by determining the effects on the patterns of reflection, successive 90 deg rotations, or both. Under these operations, the total possible number of patterns, which is 126 in this case, could be divided into 2 sets of one pattern each, 11 sets of four patterns each, and 10 sets of eight patterns each. In other words, within each set, each pattern could be produced by carrying out the abovementioned operations on any other pattern in the set. Some examples of Garner and Clement patterns from each of the three sets are shown in Figure 1. When subjects were asked to rate these dot patterns for goodness and to sort the same set of patterns according to

This research was supported by Grant BNS76-00036 from the National Science Foundation. Requests for reprints should be addressed to Allan M. Schrier, Psychology Department, Brown University, Providence, Rhode Island 02912. their similarity, subjective set size and goodness ratings agreed very well with each other and, in turn, with the objectively determined set size (Garner \& Clement, 1963). Subsequent experiments (Garner, 1974) supported Garner's hypotheses in a number of different ways: Pattern goodness was found to influence the latency of one-word naming responses, trials to criterion

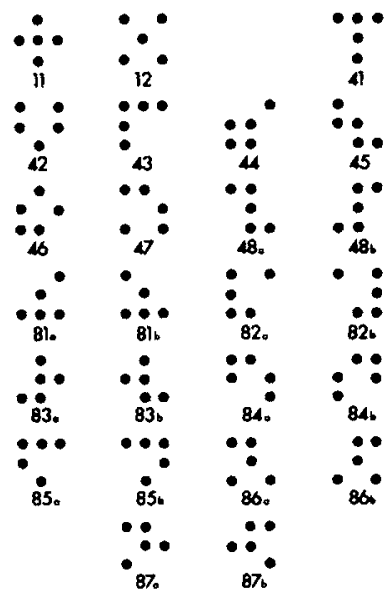

Figure 1. A sample of the Garner and Clement patterns. The first number under each pattern indicates the size of the equivalence set to which it belongs. The second number is arbitrarily assigned and simply designates the different equivalence sets of a given size. Two patterns from the same set are shown for one of the sets of size 4 and for all of the sets of size 8 . Pattern $48 \mathrm{~b}$ can only be obtained by reflection of pattern $48 \mathrm{a}$ about the horizontal or vertical axis. The second pattern in the case of each of the sets of size 8 that are illustrated represents a reflection of the first pattern about the vertical axis. Successive $90^{\circ}$ rotations of any of the patterns, except 11 and 12 , would produce additional patterns in the set. (From Clement \& Varnadoe, 1967). 
in paired-associate learning, and difficulty of discrimination in a card sorting task. Evidence to support the theory has also been obtained when using other kinds of patterns that were not composed of dots.

Clement and Weiman (1970) obtained strong evidence that the Garner and Clement patterns are processed or categorized as wholes rather than on the basis of individual elements. Citing findings by Polidora (1966), they suggested that monkeys may process individual features of patterns rather than whole patterns, and that, therefore, monkeys may not be influenced by equivalence set variables in the same way as humans are. Polidora gave rhesus monkeys two-choice discrimination problems consisting of randomly lighted cells in a pair of 4 by 4 matrices, and found that the difficulty of a problem could be predicted on the basis of the number of unique elements distinguishing a pair of patterns, that is, the number of lights that were not in corresponding cells of the two matrices.

The primary purpose of the present experiment was to test this assumption directly by giving stumptailed monkeys a series of discrimination problems composed of the Garner and Clement patterns. In the main phase of the study, a group of the animals were given a series of four types of pattern discrimination problems. The four types were as follows: a pair of patterns from different equivalence sets of size 4 (good patterns), a pair of patterns from different equivalence sets of size 8 (poor patterns), a pair of patterns from the same equivalence set of size 4 , and a pair of patterns from the same equivalence set of size 8 . The experiment was carried out in our eye-movement recording apparatus in order to test hypotheses about eye movements under these conditions. Rate of learning, choice response latency, and eye orientation were measured. If the processing of pattern information is similar to that of humans, our animals should learn problems composed of patterns drawn from small equivalence sets more quickly than they do problems composed of stimuli drawn from large equivalence sets. Also, choice response latencies and duration of individual visual fixations on the patterns should be shorter during the former type of discrimination problems than during the latter. Put simply, good patterns should be processed more quickly and be more discriminable than poor patterns. It was expected that, holding equivalence set size constant, problems composed of stimuli drawn from the same equivalence set would be more difficult and result in longer latencies and durations of fixation than problems composed of stimuli drawn from different equivalence sets. This prediction follows from the fact that stimuli from the same equivalence set are judged more similar than those drawn from different sets. The results concerning eye movements and the procedures related to recording them will be treated sparingly in the present report, since these results were largely negative.

\section{METHOD}

\section{Subjects}

Seven wild-born male stumptailed monkeys (Macaca arctoides), between 3.5 and 5 years of age, were the subjects. All animals were housed in individual cages in a colony room where they had free access to water. On test days, each animal received about $50 \%$ of its daily food ration in the form of $190-\mathrm{mg}$ banana-flavored whole-diet pellets (Noyes, Lancaster, N.H.), which were used as reinforcers in the experimental situation, with the balance of the ration, a conventional primate biscuit, fed in the home cages. The animals' weights remained constant or increased very slightly during the course of the experiment. All of the animals had had extensive training in the apparatus, including a learning set task (Schrier \& Povar, 1978) that consisted of a long series of dot-pattern discrimination problems.

\section{Apparatus}

Eye movements were measured by means of a corneal reflection procedure using an on-line computer for recording the location of the reflection and also for controlling and recording the other experimental events. The animals were tested in a modified commercial restraining chair with their heads immobilized (as required for the corneal reflection procedure) by means of a special device fitted on their heads. All of these features of the apparatus have been described before (Schrier \& Povar, 1978 , in press). During testing, the animals faced a stimulus presentation and response panel that contained three $2.5-\mathrm{cm}$ diam circular response keys, made of clear plastic with a 9-mmdiam hole in the center of each. The keys were arranged in the form of an inverted triangle, with the one at the bottom referred to as the "center key," and those at the top, the "side keys." The distance between the side keys was $8 \mathrm{~cm}$ center to center (visual angle approximately $15 \mathrm{deg}$ ) and that between each side key and the center key was $5.5 \mathrm{~cm}$ (visual angle approximately $10 \mathrm{deg}$ ). The center key was located approximately 4.3 to $5.8 \mathrm{~cm}$ below the visual axis (the exact distance varying from animal to animal because of differences in configuration of the head and placement of the head-restraining device). The discriminative stimuli were displayed on an oscilloscope, the tube face of which was mounted directly behind the three keys in the response panel. The Garner and Clement patterns were created by lighting four small dots, arranged in a square, in each of the appropriate cells of a 3 by 3 matrix. One matrix was located behind the hole in each of the side keys and each was approximately $4 \mathrm{~mm}$ square (visual angle approximately $46 \mathrm{~min})$.

\section{Procedure}

There was one test session a day, 5 days a week. Each session consisted of 300 experimental trials in addition to the 20 or 30 trials necessary to achieve initial alignment of the corneal reflection equipment. A trial was initiated when an animal pressed the center key after a small dim spot of light behind it brightened. (This aspect of the procedure was also related to calibration for purposes of eye-movement recording; see Schrier \& Povar, in press, and Schrier \& Vaughan, 1973, for details.) This center key response produced the pattern stimuli behind the side keys for a maximum of $4 \mathrm{sec}$, almost always more than enough time for a choice response. If the animal pressed the key behind which the positive pattern was displayed, a food pellet was automatically delivered, the stimuli turned off, and a $5-\mathrm{sec}$ ITI was begun. If the animal pressed the key behind which the negative pattern was displayed, the stimuli were turned off and the ITI was begun. The side on which the correct pattern appeared was randomized by sampling the state of the low order bit in a millisecond clock in the computer.

In the first (and main) phase of the experiment, five of the 
animals ( $\mathrm{Bb}, \mathrm{E}, \mathrm{F}, \mathrm{M}$, and $\mathrm{P}$ ) were given 13 blocks of four equivalence set problems, each problem consisting of two patterns. Each block consisted of one problem for which the patterns were selected at random from different equivalence sets of size 4 (D4 problems), a second problem for which the patterns were selected from different equivalence sets of size 8 (D8 problems), a third problem for which the patterns were selected from the same equivalence set of size 4 (S4) problems), and a fourth problem for which the patterns were selected from the same equivalence set of size 8 ( $\mathrm{S} 8$ problems). The order of presentation of the four types of problems within each block was randomized. The animals were trained on each problem until they reached a criterion of 19 correct responses in 20 consecutive trials within a test session.

The equivalence sets of a given size from which patterns were drawn were selected at random, as were the patterns within a set, with the restriction that all the patterns be used before any were repeated. Reuse of patterns was necessary only in the case of those from equivalence sets of size 4 and this occurred after the first 10 blocks of problems. ${ }^{2}$ No pattern was repeated if it had been used in the prior three blocks of problems. We also kept track of the distribution of problems with different numbers of unique elements in relation to the equivalence set conditions. During Phase 1 , there were 2 problems with four unique elements, which was the maximum number possible, 14 with three unique elements, 26 with two, and 10 with one. Each level of unique elements except for four (because there were so few problems in this category) was about equally represented in each level of equivalence set conditions.

The procedure was slightly different for two of the animals (Bo and C), which were tested before the others. They were given seven blocks of problems consisting of only two D4 and two D8 problems before being given six blocks consisting of the four types of problems already described, and then this was done again, except that the second time they only received 5 blocks of problems of the four types. Thus, in all, they received 11 blocks of problems of the four types. The extra blocks of problems meant that patterns had to be repeated sooner with these animals than with the other five. The performance of these two animals on the blocks of problems consisting of the four types of problems was indistinguishable from that of the other animals and only the blocks of that type were used in the data analyses.

Four of the animals ( $\mathrm{Bb}, \mathrm{E}, \mathrm{M}$, and $\mathrm{P}$ ) were given a second phase of training consisting of six blocks of problems, each block again containing the four types of problems. However, the D4 and S4 problems were composed of pairs of patterns from the six equivalence sets whose patterns had been rated by humans (Handel \& Garner, 1966) as the best of the sets of size 4 and the D8 and S8 problems were composed of patterns from the six equivalence sets whose patterns had been rated as the worst of the sets of size 8 .

Learning was measured in terms of trials to criterion, which here means the number of trials to reach the trial on which the run of 19 correct responses in 20 trials began. Choice response latency refers to the time from the onset of the pattems on the two side keys to the pressing of one of the side keys.

\section{RESULTS}

As might be expected with learning-set trained animals, there was no significant change in rate of learning of the equivalence set problems as a function of practice, and so practice effects are not considered further.

The results for Phases 1 and 2 are summarized in Table 1, which shows the mean number of trials to criterion for different size equivalence sets, that is,
Table 1

Mean Trials to Criterion (TTC) and Choice Response Latency (in Milliseconds) for Problems Composed of Patterns from Equivalence Sets of Size 4 (E4), from Sets of Size 8 (E8), from the Same Set (ES), and from Different Sets (ED) for Phases 1 and 2

\begin{tabular}{cccc}
\hline & \multicolumn{3}{c}{ Latency } \\
\cline { 3 - 4 } Condition & TTC & Pre & Crit \\
\hline \multicolumn{4}{c}{ Phase $1(\mathrm{~N}=7)$} \\
E4 & 75 & 1,089 & 922 \\
E8 & 71 & 1,066 & 906 \\
ES & 112 & 1,086 & 934 \\
& 34 & 1,064 & 894 \\
E4 & Phase 2 $(\mathrm{N}=4)$ & \\
E8 & 55 & 1,027 & 896 \\
ES & 76 & 1,034 & 854 \\
ED & 113 & 1,002 & 880 \\
& 18 & 1,060 & 870 \\
\hline
\end{tabular}

Note-Latency is shown separately for precriterion trials (Pre) and Criterion Trials (Crit) of problems.

E4 problems (D4+S4 problems) vs. E8 problems (D8+S8 problems), and for problems consisting of patterns from different as opposed to same equivalence sets, that is, ED problems (D4+D8 problems) vs. ES problems ( $44+S 8$ problems). Also shown in the table are the corresponding mean choice response latencies separated for precriterion trials of problems and for the 20 criterion trials. These results as well as the eyemovement data were analyzed by means of a series of Treatments by Subjects analyses of variance in which the main effects were equivalence set size (E4 vs. E8 problems) and same vs. different set (ES vs. ED problems). The analyses were carried out on the logarithms of the latencies and visual fixation durations. The effect of same vs. different set was highly significant for both Phase $1[F(1,6)=37.65, p<.001]$ and Phase 2 $[F(1,3)=38.43, p<.001]$, reflecting the fact that all the animals required a considerably greater number of trials to reach criterion on ES problems than on ED problems. The difference in rate of learning of problems of the two equivalence set sizes were much smaller and were not significant (Fs close to 1 in both phases). The interaction was also significant for Phase 1 $[F(1,6)=10.05, p<.025]$, reflecting the fact that the same vs. different set difference was somewhat smaller for equivalence sets of size 8 than for sets of size 4 (mean trials to criterion for D8 and S8 problems were 39 and 104, respectively, and for D4 and S4 problems, the means were 30 and 110 , respectively). There was no sign of such an interaction in Phase 2. We had expected these very large effects of the same vs. different set variable on rate of learning to be reflected both in choice response latency and aspects of eye movements, but this did not turn out to be the case. Neither of the two main equivalence set variables had a significant influence on latency during either phase of the study, nor did they consistently influence duration of visual fixations 
on the patterns or frequency of scanning (number of shifts in fixation from one pattern to the other). The distributions of these measures under the different conditions also seemed quite similar on the basis of visual inspection.

The effects on performance of the number of unique elements differentiating pairs of patterns were also analyzed for Phase 1 . Since there were very few problems involving patterns differing in four elements, such problems were eliminated from these analyses. Table 2 shows the mean trials to criterion, choice response latency, and frequency of scanning as a function of the number of unique elements. The latter two measures are shown separately for precriterion and criterion trials. A series of Treatment by Subjects analyses of variance were carried out on the data, with the main effect being number of unique elements. The increase in rate of learning with increase in number of unique elements was significant $[F(2,12)=4.49, p<.05]$. The unique elements variable also had a significant effect on $\log$ latency $[F(2,12)=6.00, p<.025]$ and amount of scanning $[F(2,12)=15.20, p<.001]$ during precriterion trials of problems but not during criterion trials. For both latency and amount of scanning, Newman-Keuls tests indicated significant differences (all ps $<.05$ ) between problems involving one and two and one and three unique elements, but not between those involving two and three. Thus, in terms of eye movements, differences in duration of individual fixations on each pattern had little to do with the relatively short latencies on problems with the fewest number of unique elements, but rather the animals tended to look back and forth at the two patterns less frequently on these problems.

Although the experiment was not designed specifically for the purpose, the use of problems involving stimuli from the same equivalence set also gave us the opportunity to analyze possible differences in effects of rotations and reflections of different kinds and degrees, something which has been of interest in prior studies with animals (Riopelle, Rahm, Itoigawa, \& Draper, 1964) and with humans (Royer \& Holland, 1975). Our initial analysis involved only the S8 problems, because only in these is one pattern solely a re-

Table 2

Mean Trials to Criterion (TTC), Choice Response Latency (in Milliseconds), and Frequency of Scans per Trial as a Function of the Number of Unique Elements Between Pairs of Patterns Presented During Phase 1

\begin{tabular}{|c|c|c|c|c|c|}
\hline \multirow{2}{*}{$\begin{array}{l}\text { Unique } \\
\text { Elements }\end{array}$} & \multirow{2}{*}{ TTC } & \multicolumn{2}{|c|}{ Latency } & \multicolumn{2}{|c|}{ Scans } \\
\hline & & Pre & Crit & Pre & Crit \\
\hline $\begin{array}{l}1 \\
2 \\
3\end{array}$ & $\begin{array}{r}100 \\
72 \\
56\end{array}$ & $\begin{array}{l}1,014 \\
1,124 \\
1,100\end{array}$ & $\begin{array}{l}888 \\
914 \\
937\end{array}$ & $\begin{array}{l}1.19 \\
1.42 \\
1.37\end{array}$ & $\begin{array}{l}0.94 \\
1.06 \\
1.09\end{array}$ \\
\hline
\end{tabular}

Note-The latency and scans data are presented separately for precriterion (Pre) and criterion (Crit) trials of problems. flection or rotation of the other. In virtually all S4 problems, one pattern is both a reflection and a rotation of the other. ${ }^{2}$ The $\mathbf{S} 8$ problems that were given to the five animals that had Phases 1 and 2 were separated into four types: those in which one member of the pair was (1) a 90 deg rotation of the other, (2) a 180 deg rotation of the other, (3) an orthogonal reflection of the other (that is, a left-right or up-down reflection), or (4) a reflection of the other about either diagonal axis. (There were three to six problems of each type, the number depending on the animal.) The trials to criterion means for the four types of problems were, respectively, $100,95,196$, and 115 . The effect of these four types of S8 problems on rate of learning was significant $[F(3,12)$ $=8.68, \mathrm{p}<.01]$ in a Treatment by Subjects analysis of variance. The Newman-Keuls test indicated that only the mean for orthogonal reflections was significantly different from the means for the other types of problems (all ps $<.01$ ). There were no corresponding differences in either latency or eye movements. Analysis of S4 problems produced virtually identical results $[F(3,12)=$ $6.74, p<.01]$. This outcome, taken together with that for $\mathbf{S 8}$ problems, suggests that orthogonal reflection dominates in determining discriminability when one of the stimuli can be produced either by such a reflection or by rotation. There have been indications in the previous literature that left-right reflections (reflections about the vertical axis) are more difficult than are up-down (reflections about the horizontal axis) for both humans and monkeys, at least when the patterns are presented in the vertical plane (Royer \& Holland, 1975). However, when we divided the problems that involved orthogonal reflections into the two types, the difference between them in terms of rate of learning was very small and did not approach significance.

\section{DISCUSSION}

In contrast to humans (Garner, 1974), our monkeys did not find patterns from equivalence sets of size 4 easier to process than those from equivalence sets of size 8. However, as is the case with humans, our monkeys did find patterns from the same equivalence set less discriminable than those from different sets. The fact that this last outcome is independent of the number of unique elements distinguishing the patterns suggests that our failure to find an effect of size of equivalence set, that is, of pattern goodness in Garner's terms, was not a matter of the monkeys responding primarily to features as opposed to whole patterns. This, of course, is not to say that they were not influenced by features, for there was clear evidence in the present study, as there was in Polidora's (1966), that the greater the number of unique elements distinguishing a pair of patterns composing a problem, the faster the discrimination learning. As it turns out, however, this finding is not restricted to monkeys. A recent experi- 
ment carried out in Garner's laboratory (Smith, Note 1), in which humans were given a speeded card-sorting task involving the Garner and Clement patterns, showed a significant effect of the number of unique elements in addition to the usual effects of set size and same vs. different sets.

We have no explanation for our failure to find the very striking effects of same vs. different set reflected in either choice response latency or eye movements. It is certainly not a matter of these dependent variables being relatively insensitive in the present experimental context, because we have found large changes in these measures in several discrimination learning studies using the same apparatus (Geary \& Schrier, 1975; Schrier \& Povar, 1978). Also, significant changes in latency and amount of scanning were found in the present study as a function of the number of unique elements. The differences in average latency in some of the studies with humans have been rather small (Pomerantz, 1977), as they were here, but the number of subjects was usually larger than in the present study, which perhaps accounts for some of our failures to find significant differences.

Contrary to what might be expected, latency was shortest and amount of scanning least frequent for problems with the fewest number of unique elements. Since this occurred only for precriterion trials, it might reflect an initial tendency for the animals to respond precipitously because of the high degree of similarity of the positive and negative stimuli.

There have been a number of studies with animals concerned with effects of reflection and rotation of the stimuli on discriminative performance, but in few has the problem been approached systematically. Some aspects of the results reported by Riopelle et al. (1964), who presented letter-like line patterns to rhesus monkeys, are equivalent to our finding that ES problems were harder than ED problems, though the differences were much greater in our case. Hunton and Hicks (1965) reported evidence which suggested that discriminations involving $180 \mathrm{deg}$ rotations were substantially more difficult for both monkeys and young children than those involving dissimilar stimuli, but the experiment was not designed to gauge the effects precisely.

Our findings do not agree with those of Riopelle et al. for finer analyses of rotation and reflection effects. They found that $90 \mathrm{deg}$ rotations were significantly more difficult than orthogonal reflections taken as a whole, quite the opposite of our findings on the question. Also, as mentioned earlier, Riopelle et al. found, as have some others when the stimuli were presented in the vertical plane (Royer \& Holland, 1975), that reflections about the vertical axis are more difficult than those about the horizontal, whereas they were about equally difficult in the present study. Riopelle et al. also reported that problems involving the latter type of reflections were easier than even their ED problems. However, for none of the types of reflection and rotation that we analyzed was rate of learning close to that on ED problems. This suggests that simple accounts of the effects of same vs. different sets in terms of preference for observing one or another quadrant or segment of the stimuli (Riopelle et al., 1964) are not appropriate. Some of the differences in results of the two studies may be traceable to the use of a less systematic sampling of set sizes, number of sets, and stimuli from within sets in the Riopelle et al. study than in the present one.

There are two major conclusions that we can draw on the basis of the present experiment. The first is that, at least as judged by the discriminability of the patterns, there are both similarities and differences in the way in which monkeys and humans process patterns of the type considered here. The second is that for both species of subjects the processing seems more complicated than some have suggested (Garner, 1974; Polidora, 1966), in that it is not specifiable either in terms of elements of the patterns or in terms of some factor related to whole patterns, but seems under the same circumstances to be a matter of both.

\section{REFERENCE NOTE}

1. Smith, A. F. Holistic and feature-analytic aspects of multidimensional pattern recognition. Unpublished manuscript, 1976.

\section{REFERENCES}

Clement, D. E., \& Varnadoe, K. W. Pattern uncertainty and the discrimination of visual patterns. Perception \& Psychophysics, 1967, 2, 427-431.

Clement, D. E., \& Weiman, C. F. R. Instructions, strategies, and pattern uncertainty in a visual discrimination task. Perception \& Psychophysics, 1970, 7, 333-336.

GARNER, W. R. Uncertainty and structure as psychological concepts. New York: Wiley, 1962.

GARNER, W. R. The processing of information and structure. Hillsdale, N.J: Erlbaum, 1974.

GaRner, W. R., \& Clement, D. E. Goodness of pattern and pattern uncertainty. Journal of Verbal Leaming and Behavior, $1963,2,446-452$.

Geary, N. D., \& Schrier, A. M. Eye movements of monkeys during performance of ambiguous cue problems. Animal Leaming \& Behavior, 1975, 3, 167-171.

HANDEL, S., \& Garner, W. R. The structure of visual pattern associates and pattern goodness. Perception \& Psychophysics, 1966, 1, 33-38.

Hunton, V. D., \& Hicks, L. H. Discrimination of figural orientation by monkeys and children. Perceptual and Motor Skills, 1965, 21, 55-59.

Polrdora, V. J. Stimulus correlates of visual pattern discrimination by monkeys. Multidimensional analyses. Perception \& Psychophysics, 1966, 1, 405-414.

Pomerantz, J. R. Pattern goodness and speed of encoding. Memory \& Cognition, 1977, 5, 235-241.

Riopelle, A. J., Rahm, U., Itoigawa, N., \& Draper, W. A. Discrimination of mirror-image patterns by rhesus monkeys. Perceptual and Motor Skills, 1964, 19, 383-389. 
ROYER, F. L., \& Holland, T. R. Rotational transformation of visual figures as a clinical phenomenon. Psychological Bulletin, 1975, 82, 843-868.

Schrier, A. M., \& Povar, M. L. Eye movements of monkeys during learning set formation. Science, 1978, 199, 1362-1364.

Schrier, A. M., \& Povar, M. L. Eye movements of stumptailed monkeys during discrimination learning: VTE revisited. Animal Learning \& Behavior, in press.

SChrier, A. M., \& VAUghaN, J. Eye movements of monkeys during learning of color and form discrimination problems involving reversal and nonreversal shifts. Primates, 1973, 14, 161-178.

\section{NOTES}

1. We used 10 of the 11 equivalence sets of size 4 and 9 of the 10 sets of size 8 . The patterns in the eliminated sets were in each case the same as those in another set of the same size except that they were displaced one cell horizontally or vertically. Samples of patterns from the sets in question are the patterns labeled $4 \mathrm{e}$, $4 f, 8 a$, and $8 b$ in Figure 1 in Handel and Garner, 1966.

2. For example, the pattern labeled 42 in Figure 1 can be reflected about its horizontal axis to produce another pattern in its set. This pattern can also be produced by a $180^{\circ}$ rotation of the original pattern. This is merely another way of saying that the equivalence sets of size 4 are more redundant than those of size 8 . In analyzing the 54 patterns, as mentioned later in the paragraph, a problem composed of the patterns just mentioned would be included in both the types of problems that involved $180^{\circ}$ rotations and those that involved orthogonal reflections.

(Received for publication August 11, 1978; revision accepted December $8,1978$. 